\title{
Estudio de percepción de las metodologías de enseñanza - aprendizaje en un postgrado de ingeniería
}

\author{
Mercedes Valdivieso Bolaños ${ }^{1}$ \\ Carolina Montoya Rodríguez ${ }^{2}$ \\ Paloma Martínez Sánchez ${ }^{3}$
}

Recibido: 12-09-2018

Aceptado: 03-12-2018

\section{Resumen}

Para las instituciones de educación superior es importante conocer la calidad de su servicio para mejorar sus procesos. El presente estudio busca identificar la percepción de los estudiantes de una especialización de ingeniería sobre las metodologías de enseñanza-aprendizaje. Esta investigación se desarrolló a través de una encuesta a 3 grupos de estudiantes de diferentes semestres. El estudio arrojó que las metodologías de enseñanza-aprendizaje más empleadas por los docentes son: la clase magistral (100\%) y el aprendizaje cooperativo (77\%). Más del $80 \%$ de estudiantes percibe que las metodologías son suficientes y apropiadas, mientras que menos del $50 \%$ de estudiantes las percibe como insuficientes

\footnotetext{
1. Administradora de Obras Civiles de la Fundación Universitaria del Área Andina y Especialista en Gerencia de Producción y Productividad de la Universidad El Bosque. Directora de la Planta de Triturados Centrasa sede Tolima. CONSTRUCTORA LHS S.A.S.

Tolima - Colombia.

2. Diseñadora Industrial de la Universidad de Los Andes. Maestría en Ciencia Holística de la Universidad de Plymouth, Inglaterra. Profesora Asociada del programa de Ingeniería Industrial de la Universidad El Bosque.

Bogotá - Colombia.

Correo electrónico: montoyacarolina@unbosque.edu.co

ORCID: https://orcid.org/0000-0002-5425-591X

Google academico: https://scholar.google.com/citations?user=JXj80z4AAAAJ\&hl=es
}

3. Ingeniera de Producción Agroindustrial Universidad de La Sabana. Especialización en Gerencia Estratégica y Competitiva Universidad de La Sabana. Maestra en Ciencias con énfasis en Sistemas de Calidad y productividad del Instituto Tecnológico de Monterrey -México. Doctora en Logística y Cadena de Suministro de la Universidad Popular Autónoma del Estado de Puebla (UPAEP). Profesora Titular del programa de Ingeniería Industrial, Universidad El Bosque.

Bogotá - Colombia.

Correo electrónico: martinezpaloma@unbosque.edu.co 
e inadecuadas. Se concluye que hay una estrecha relación entre la evaluación de las metodologías impartidas por los docentes y la adquisición y aplicación del conocimiento aprendido por los estudiantes, por lo cual es importante promover la aplicación de las metodologías mejor evaluadas para mejorar la calidad del servicio.

Palabras clave: proceso enseñanza - aprendizaje; metodología; percepción; gerencia de producción y productividad.

\title{
Perception study of learning teaching methodologies in an engineering postgraduate
}

\begin{abstract}
For the Institutions of Higher Education it is important to know the quality of its service to improve its processes. The present study seeks to identify the students' perception of an engineering specialization on Teaching-Learning Methodologies. This research was developed through a survey of 3 groups of students from different semesters. The study showed that the Teaching-Learning Methodologies most used by teachers are: Lecture (100\%) and Cooperative Learning (77\%). More than $80 \%$ of students perceive that methodologies are sufficient and appropriate, while less than $50 \%$ of students perceive them as insufficient and inadequate. It is concluded that there is a close relationship between the evaluation of the methodologies taught by the teachers and the acquisition and application of the knowledge learned by the students, which is why it is important to promote the application of the best evaluated methodologies to improve the quality of the service.
\end{abstract}

Keywords: Teaching - Learning Process; Methodology; Perception; Management of Production and Productivity. 


\title{
Estudo de Percepção de Metodologias de Ensino de Aprendizagem em uma Pós- Graduação em Engenharia
}

\begin{abstract}
Resumo
Para as Instituições de Ensino Superior é importante conhecer a qualidade de seus serviços para melhorar seus processos. O presente estudo busca identificar a percepção dos estudantes sobre uma especialização em engenharia nas metodologias de ensinoaprendizagem. Esta pesquisa foi desenvolvida através de uma pesquisa com 3 grupos de estudantes de diferentes semestres. $O$ estudo mostrou que as metodologias de ensino-aprendizagem mais utilizadas pelos professores são: Palestra (100\%) e Aprendizagem Cooperativa (77\%). Mais de $80 \%$ dos estudantes percebem que as metodologias são suficientes e apropriadas, enquanto menos de $50 \%$ dos estudantes as percebem como insuficientes e inadequadas. Conclui-se que existe uma estreita relação entre a avaliação das metodologias lecionadas pelos professores e a aquisição e aplicação do conhecimento aprendido pelos alunos, motivo pelo qual é importante promover a aplicação das melhores metodologias avaliadas para melhorar a qualidade do serviço.
\end{abstract}

Palavras-chave: Ensino - Processo de Aprendizagem; Metodologia; Percepção; Gestão de Produção e Produtividade.

\section{Introducción}

El aprendizaje, según Piaget, es un proceso mediante el cual el sujeto, a través de la experiencia, la manipulación de objetos, la interacción con las personas, genera o construye conocimiento del mundo que los rodea, mediante el proceso de asimilación y acomodación (Martin, 2003). Las metodologías del aprendizaje se definen como las distintas modalidades de enseñanza -apren- 
dizaje (presenciales, semipresenciales) y los diferentes métodos que el docente selecciona para lograr este proceso (Pérez, 2014, p. 22). La enseñanza se refiere a la facilitación del aprendizaje. Esta labor es desarrollada por el profesor, e involucra variables como: su propia personalidad, una sólida preparación en el campo de su profesión, y recursos pedagógicos que le permiten facilitar el aprendizaje en sus estudiantes (Zarate, 2002, p. 19).

El diseño curricular debe tener en cuenta la definición de los objetivos, de contenidos y la manera de planificar las actividades de aprendizaje para que se ajusten a las necesidades del estudiante (Coll, 1987). La enseñanza eficaz es la que parte del nivel de desarrollo del estudiante, "pero no para acomodarse a él, sino para hacerlo progresar a través de su Zona de Desarrollo Próximo" (Núñez, 2015, p. 85). Un aspecto importante a tener en cuenta para que el estudiante tenga un aprendizaje significativo es lograr que el nuevo contenido de aprendizaje tenga un significado cercano a los esquemas del estudiante para que el proceso de enseñanza-aprendizaje sea fluido (Núñez, 2015, p. 83).

Según el profesor De Miguel (2006), la planeación didáctica de una materia debe estar orientada a la adquisición de las competencias (aprendizajes) propuestos para los estudiantes, a través del diseño y la selección de "escenarios, metodologías y modalidades de trabajo para profesores y estudiantes, que conduzcan de manera eficaz a las metas propuestas". Según lo anterior, a continuación, se presentan las Metodologías de EnseñanzaAprendizaje tenidas en cuenta para el presente estudio:

Enseñanza Por Procesos (EPP). "Descansa en el paradigma mediacional del proceso de enseñanza - aprendizaje, donde el aprendizaje es el resultado de la interacción de los individuos en el entorno sociocultural en el cual se desarrollan" (García \& Galicia, 2014, p. 21).

Aprendizaje Cooperativo (AP). Los estudiantes trabajan divididos en pequeños grupos en actividades de aprendizaje (división de rendimiento por equipos, torneo de juegos por equipos, 
equipos de investigación) y son evaluados según la productividad del grupo. Se puede considerar como un método a utilizar entre otros o como una filosofía de trabajo (Suárez, 2010).

Aprendizaje Basado en Proyectos (ABP). El producto del proceso de aprendizaje es un proyecto o programa de intervención profesional, en torno al cual se articulan todas las actividades formativas (Maldonado, 2008).

Contrato de Aprendizaje (CA). Existe un contrato didáctico o de aprendizaje cuando estudiante y profesor de forma explícita intercambian sus opiniones, comentan sus necesidades, sus sentimientos, comparten proyectos y deciden en colaboración la forma de llevar a cabo el proceso de enseñanza y aprendizaje y lo reflejan oralmente o por escrito (García \& Bagán, 2006).

Aprendizaje Basado en Problemas (ABP). La base es un problema para identificar temas de aprendizaje para su estudio grupal y/o individual. La pregunta es el detonador para que los estudiantes cubran los objetivos de aprendizaje en curso. Se organizan e integran temas con base a problemas de la vida real, en donde confluyen diversas áreas del conocimiento y se realizan diversas actividades para la solución del problema (García \& Galicia, 2014).

Clase Magistral (CM). Presentar de manera organizada información (profesor-alumnos), activar la motivación y procesos cognitivos. Entre las actividades están: Presentación de los contenidos de forma verbal y fijación de los contenidos, por medio de ejercicios complementarios y tareas (Ropero, García, \& Diez, 2013).

Estudio de Casos (EC). Es un proceso de enseñanza-aprendizaje donde el sujeto se enfrenta ante la descripción de una situación real y específica, que debe ser considerada en una amplia discusión para la toma de decisiones. El fin es llegar a una conceptualización experiencial y realizar una búsqueda de soluciones eficaces y toma de decisiones (García \& Galicia, 2014). 
Simulación y Juego (SJ). "Consiste en situar a un estudiante en un contexto que imite algún aspecto de la realidad y en establecer en este ambiente situaciones, problemáticas o reproductivas similares a las que él tendría que enfrentar en situaciones reales" (Andreu, García, \& Mollar, 2005).

Para un análisis de la enseñanza universitaria es importante conocer la opinión de los miembros que la constituyen, y especialmente la de los estudiantes que son el motivo de existencia de las entidades educativas. Por tal razón surge el presente estudio de percepción de los estudiantes respecto a las metodologías de enseñanza aprendizaje impartidas en la especialización de Gerencia de Producción y Productividad, con el propósito que los docentes utilicen las metodologías adecuadas para que el estudiante pueda lograr las competencias propuestas como meta de aprendizaje (Aparicio \& Ostos, 2018). En ese sentido, el estudio parte del interrogante ¿Según la percepción de los estudiantes de la especialización en Gerencia de Producción y Productividad, las metodologías de enseñanza aprendizaje que emplean los docentes para que los alumnos adquieran un aprendizaje significativo, son suficientes y apropiadas?

\section{Metodología}

La metodología de la Investigación se estructuró de 5 fases: 1. Exploración; 2. Población y muestra; 3. Diseño y desarrollo del instrumento de recolección de datos; 4 . Aplicación de encuestas; 5. Tabulación y análisis de datos, explicadas a continuación.

- Exploración: se investigaron diferentes autores para indagar sobre las metodologías de enseñanza-aprendizaje existentes, según la información obtenida se encontraron 8 de las más nombradas por la literatura (de Almeida, Santos, Porto, 2017) y con esta información se diseñó una encuesta para ser diligenciada por los docentes de las di- 
ferentes materias de la especialización y definir cuáles de estas metodologías son las más empleadas por ellos. Y con estas diseñar la encuesta para los estudiantes.

- Población y muestra: la muestra está representada por los estudiantes de especialización vinculados en el segundo semestre del 2013 y el primer y segundo semestre del 2014. La selección de la muestra será aleatorio probabilístico simple. Los estudiantes encuestados son 55; el $45 \%$ (25) de ellos son los vinculados en el segundo semestre del 2014, el 36\% (20) en el primer semestre del 2014 y el $18 \%$ (10) para los vinculados en el segundo semestre del 2013. Se realizaron 45 encuestas presenciales correspondientes a los alumnos vinculados en el año 2014 y 10 encuestas por correo electrónico de los alumnos vinculados en el segundo semestre del 2013.

- Diseño y desarrollo del instrumento de recolección de datos: El Instrumento de medida y recolección de datos es un test llamado "Evaluación sobre las metodologías aplicadas en la Especialización de Gerencia de Producción y Productividad", el cuestionario contempla 39 preguntas, que evalúan las metodologías de enseñanza aprendizaje empleadas en las 13 materias que se dictan en los cursos de la especialización. En esta encuesta se tienen en cuenta los objetivos de cada materia, los cuales fueron tomados del Syllabus de los cursos de especialización.

- Aplicación de encuestas: El test se envía por medio electrónico y también se suministra en forma presencial, con la colaboración de las directivas de la Institución. Hay tres tipos de preguntas: La primera, evalúa las metodologías de aprendizaje empleadas en la especialización. Tiene escala numérica de 5 posiciones donde el puntaje más alto será 5 y el más bajo 1. Las respuestas a esta primera pregunta se califican de la siguiente manera: entre 4.5 y 5 excelente, entre 4.4 y 4 bueno, entre 3 y 3.9 aceptable, 
entre 2 y 2.9 regular y menos de 2 malo. La segunda, evalúa si las metodologías son suficientes y apropiadas. Es complemento de la primera, es de afirmación positiva o negativa con explicación de la negación. Y la tercera, evalúa si actualmente son aplicados los conocimientos adquiridos en las materias. Es de afirmación positiva o negativa con explicación de cada afirmación.

- Plan de tabulación y análisis de datos: La calidad de la información se valoró en términos de pertinencia, relevancia y de fiabilidad y validez con el análisis de Cronbach o análisis factorial, este análisis se realizó a cada materia, se calculó la varianza a los resultados de los puntajes de las metodologías y con estos resultados se calculó el coeficiente de Cronbach con la siguiente formula:

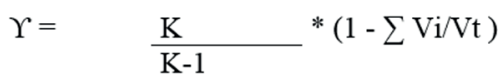

Dónde: $\mathrm{K}=$ número de ítems

$\mathrm{Vi}=$ varianza de cada ítem

$\mathrm{Vt}=$ Varianza de la suma de los Items

Obteniéndose una fiabilidad mínima del $0.83 \%$. Mayor 0.80 que la establecida por Cronbach para que los resultados sean fiables. Para el análisis de los datos obtenidos se utilizan técnicas estadísticas descriptivas con el propósito de determinar porcentajes e incidencias de las mismas y sus respectivas agrupaciones y tendencia.

\section{Resultados}

La especialización en Gerencia de Producción y Productividad consta de 13 materias, éstas son: 1. Metodología de la Investigación, 2. Gerencia de Producción y Calidad, 3. Modelos Operacionales, 4. Gerencia de Recursos Humanos, 5. Logística de distribución, 6. Gerencia Estratégica de Producción, 7. Sistematización de 
la Producción, 8. Juego Gerencial, 9. Gestión de Costos y Métodos de Inventarios, 10. Innovación y Tecnología, 11. Salud Ocupacional, Seguridad y Salud en el Trabajo, 12. Informática Industrial, y 13. Robótica y Telecomunicaciones.

\section{Metodologías de enseñanza-aprendizaje empleadas por los docentes de la especialización.}

De las 8 metodologías de enseñanza-aprendizaje investigadas para el presente estudio, los docentes de la especialización solo aplican 6: 1. Exposición Magistral, 2. Aprendizaje Cooperativo, 3. Estudio de Casos, 4. Aprendizaje Basado en Proyectos (ABP), 5. Aprendizaje Basado en Problemas, y 6 . Simulación y Juego, como muestra la Tabla 1. Las metodologías no utilizadas por los docentes son: Contrato de Aprendizaje y Enseñanza Por Procesos.

Tabla 1. Metodologías de enseñanza - aprendizaje empleadas por los docentes en la especialización.

\begin{tabular}{|c|c|c|c|c|c|c|}
\hline Materia & $\begin{array}{l}\text { Exposición } \\
\text { Magistral }\end{array}$ & $\begin{array}{l}\text { Aprendizaje } \\
\text { Cooperativo }\end{array}$ & $\begin{array}{l}\text { Estudio de } \\
\text { Casos }\end{array}$ & $\begin{array}{l}\text { Aprendizaje } \\
\text { Basado en } \\
\text { Proyectos } \\
\text { (ABP) }\end{array}$ & $\begin{array}{l}\text { Aprendizaje } \\
\text { Basado en } \\
\text { Problemas }\end{array}$ & $\begin{array}{l}\text { Simulación } \\
\text { y } \\
\text { Juego }\end{array}$ \\
\hline $\begin{array}{l}\text { Metodología de la } \\
\text { Investigación }\end{array}$ & $x$ & $x$ & & & & \\
\hline $\begin{array}{l}\text { Gerencia de } \\
\text { Producción y } \\
\text { Calidad }\end{array}$ & $x$ & & $x$ & $\mathrm{x}$ & & \\
\hline $\begin{array}{l}\text { Modelos } \\
\text { Operacionales }\end{array}$ & & & & $x$ & & $x$ \\
\hline $\begin{array}{l}\text { Gerencia de } \\
\text { Recursos Humanos }\end{array}$ & $x$ & $x$ & $x$ & & & \\
\hline $\begin{array}{l}\text { Logística de } \\
\text { distribución }\end{array}$ & $x$ & $x$ & $x$ & & & \\
\hline $\begin{array}{l}\text { Gerencia } \\
\text { Estratégica de } \\
\text { Producción }\end{array}$ & $x$ & $x$ & $x$ & & & \\
\hline $\begin{array}{l}\text { Sistematización de } \\
\text { la Producción }\end{array}$ & $x$ & & & $x$ & $\mathrm{x}$ & $x$ \\
\hline Juego Gerencial & $x$ & $x$ & $x$ & & & \\
\hline $\begin{array}{l}\text { Gestion de Costos } \\
\text { y Métodos de } \\
\text { Inventarios }\end{array}$ & $x$ & $x$ & & & & \\
\hline $\begin{array}{l}\text { Innovación y } \\
\text { tecnogogía }\end{array}$ & $x$ & $x$ & & $x$ & & \\
\hline
\end{tabular}




\begin{tabular}{|c|c|c|c|c|c|c|}
\hline Materia & $\begin{array}{l}\text { Exposición } \\
\text { Magistral }\end{array}$ & $\begin{array}{l}\text { Aprendizaje } \\
\text { Cooperativo }\end{array}$ & $\begin{array}{l}\text { Estudio de } \\
\text { Casos }\end{array}$ & $\begin{array}{l}\text { Aprendizaje } \\
\text { Basado en } \\
\text { Proyectos } \\
\text { (ABP) } \\
\end{array}$ & $\begin{array}{l}\text { Aprendizaje } \\
\text { Basado en } \\
\text { Problemas }\end{array}$ & $\begin{array}{l}\text { Simulación } \\
\text { y } \\
\text { Juego }\end{array}$ \\
\hline $\begin{array}{l}\text { Salud Ocupacional } \\
\text { Seguridad y Salud } \\
\text { en el } \\
\text { Trabajo }\end{array}$ & $x$ & $x$ & $x$ & & $x$ & \\
\hline $\begin{array}{l}\text { Informática } \\
\text { Industrial }\end{array}$ & $x$ & $x$ & $x$ & & & \\
\hline \multirow[t]{2}{*}{$\begin{array}{l}\text { Robótica y } \\
\text { Telecomunicaciones }\end{array}$} & $x$ & $x$ & & $x$ & & \\
\hline & 13 & 10 & 7 & 5 & 2 & 2 \\
\hline $\begin{array}{l}\text { Porcentaje de } \\
\text { docentes }\end{array}$ & $100 \%$ & $77 \%$ & $54 \%$ & $38 \%$ & $15 \%$ & $15 \%$ \\
\hline
\end{tabular}

Fuente. Elaboración propia.

En la especialización se implementan principalmente 2 metodologías de enseñanza aprendizaje: La primera, es la clase magistral utilizada por un $100 \%$ de los docentes, esto significa que se utiliza en las 13 materias impartidas en el curso. Su uso es básicamente porque los docentes tienen que exponer de alguna manera la teoría y conceptos que sustentan la temática a desarrollar en cada materia. El porcentaje de tiempo que le dedican los docentes a esta metodología varía para cada materia, porque en el desarrollo de la clase los docentes emplean varias metodologías, por ejemplo, explican los conceptos durante 20 minutos en una clase magistral y en el resto del tiempo hacen talleres. La segunda, el Aprendizaje Cooperativo empleado por el $77 \%$ de los docentes. Esta es utilizada por docentes de 10 de las materias de la especialización. Las actividades más utilizadas por esta metodología son talleres y trabajos en grupo. Los talleres son muy acogidos por los estudiantes porque afianzan el conocimiento (Langer, 2016).

Las restantes metodologías de enseñanza aprendizaje empleadas por los docentes son:

Estudio de casos, aplicada por el $54 \%$ de los docentes, utilizada en las materias Gerencia de Producción y Calidad, Gerencia de Recursos Humanos, Logística de Distribución, Gerencia Estratégica de Producción, Juego Geren- 
cial, Salud Ocupacional Seguridad y Salud en el trabajo e Informática Industrial. Esta metodología es empleada por las materias relacionadas con administración y gerencia porque se desarrolla la capacidad de toma de decisiones. Las materias que no la emplean son aquellas relacionadas con la utilización de un software y sistemas como son: Modelos Operacionales, Sistematización de la Producción y Robótica y Telecomunicaciones.

Aprendizaje Basado en Proyectos (ABP), utilizada en un $38 \%$ por los docentes, en las materias de: Gerencia de Producción y Calidad, Modelos Operacionales, Sistematización de la Producción e Innovación y Tecnología. En las 4 materias consistió en desarrollar un proyecto con la identificación y solución de un problema real de las empresas donde laboran los estudiantes. Esta es una metodología muy activa y se logra un aprendizaje significativo porque se parte de la experiencia de los estudiantes de varias disciplinas, se aplica el conocimiento adquirido en la materia y se elabora un nuevo conocimiento (Aparicio, 2018).

Aprendizaje Basado en Problemas (ABP), utilizada por el $15 \%$ de los docentes, empleada en la materia de Sistematización de la Producción y Salud Ocupacional Seguridad y Salud en el trabajo. En las dos materias el docente planteó los problemas para que los estudiantes los solucionaran y debatieran.

Y Simulación y Juego, empleada por 15\% de los docentes, en las materias de Modelos Operacionales y Sistematización de la Producción. Se emplean en estas materias porque están relacionadas con la utilización de un software para simular operaciones y procesos productivos. 


\section{Percepción de los estudiantes respecto a las metodologías de enseñanza-aprendizaje de la especialización.}

Con base en el objetivo de aprendizaje de cada una de las materias los estudiantes evaluaron las metodologías de aprendizaje empleadas en las 13 materias de la especialización, por cada docente.

En una primera instancia los estudiantes evaluaron si las metodologías de enseñanza aprendizaje implementadas en cada clase son excelentes (4.5-5.0); buenas (4.4-4.0); aceptables (3.0-3.9); regulares (2.0-2.9); y malas (menos de 1.9). En la tabla 2 se hace un resumen de los puntajes obtenidos por metodología en cada materia. El 1.0 corresponde al menor puntaje y el 5.0 al mayor. Las materias que están en blanco corresponden a las que fueron evaluadas como buenas y excelentes, las materias en gris corresponden a las que fueron evaluadas como aceptables y finalmente las que están en negro corresponden a las que fueron evaluadas como regulares.

La materia que obtuvo los mayores puntajes fue Gestión de Costos y Métodos de Inventarios con 4.3 para la Clase Magistral y 4.4 para el Aprendizaje Cooperativo, el menor puntaje fue para Metodología de la Investigación con 2.3 para la Clase Magistral y 2.6 para el Aprendizaje Cooperativo como se evidencia en la tabla 2. 


\begin{tabular}{|c|c|c|c|c|c|c|c|c|c|c|c|c|c|c|}
\hline $\begin{array}{l}\text { Metodologías } \\
\text { / Materias }\end{array}$ & 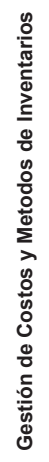 & 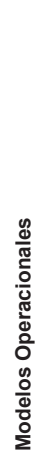 & 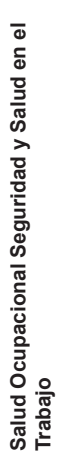 & 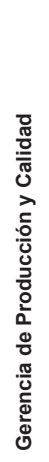 & 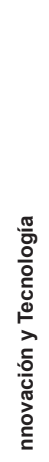 & 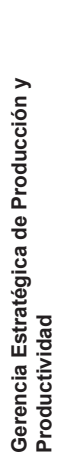 & 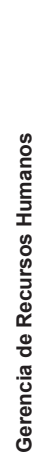 & 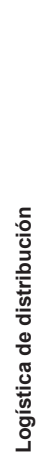 & 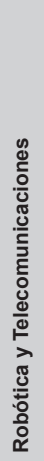 & 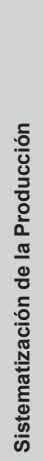 & 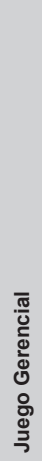 & 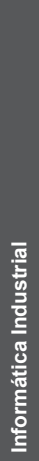 & 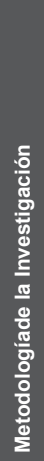 & 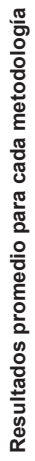 \\
\hline $\begin{array}{l}\text { Clase } \\
\text { Magistral }\end{array}$ & 4,3 & 4,3 & 4,3 & 4,0 & 4,0 & 4,1 & 4,0 & 3,8 & 3,4 & 3,5 & 3,7 & 3,1 & 2,3 & 3,8 \\
\hline $\begin{array}{l}\text { Aprendizaje } \\
\text { Cooperativo }\end{array}$ & 4,4 & & 4,2 & & 4,4 & 4,0 & 3,9 & 3,9 & 3,6 & 3,6 & 3,6 & 3,1 & 2,6 & 3,8 \\
\hline $\begin{array}{l}\text { Estudio de } \\
\text { Casos }\end{array}$ & & & & 4,3 & & 3,8 & 3,8 & 3,9 & & & 3,3 & 2,8 & & 3,7 \\
\hline $\begin{array}{l}\text { Aprendizaje } \\
\text { Basado en } \\
\text { Proyectos } \\
\text { ABP }\end{array}$ & & 4,2 & & 4,2 & 4,2 & & & & 3,7 & & & & & 4,1 \\
\hline $\begin{array}{l}\text { Aprendizaje } \\
\text { Basado en } \\
\text { Problemas }\end{array}$ & & & 4,0 & & & & & & & & & & & 4,0 \\
\hline $\begin{array}{l}\text { Simulación y } \\
\text { Juego }\end{array}$ & & 4,4 & & & & & & & & 3,4 & & & & 3,9 \\
\hline $\begin{array}{l}\text { Resultados } \\
\text { promedio } \\
\text { para } \\
\text { cada materia }\end{array}$ & 4,4 & 4,3 & 4,2 & 4,2 & 4,2 & 4,0 & 3,9 & 3,9 & 3,6 & 3,5 & 3,5 & 3,0 & 2,5 & \\
\hline
\end{tabular}

Tabla 2. Evaluación de las metodologías de enseñanza-aprendizaje empleadas en cada materia según los objetivos de aprendizaje planteados.

Se observa que entre las metodologías más aceptadas por los estudiantes están: Aprendizaje Basado en Proyectos ABP con un puntaje promedio de 4.1 y aprendizaje Basado en Problemas ABP con un puntaje promedio de 4.0. Las metodologías que obtuvieron un puntaje promedio menor que 4.0 fueron Simulación y Juego, Clase Magistral, Aprendizaje Cooperativo y estudio de casos. En cuanto a estudio de casos que obtuvo el menor puntaje promedio de 3.7, algunos estudiantes opinan que es una buena metodología pero que los casos deberían ser aplicados a la realidad colombiana.

Luego los estudiantes evaluaron si las metodologías son suficientes y apropiadas para adquirir y aplicar el conocimiento de cada 
materia (Ortiz, Buitrago, 2017). En la gráfica 1 se muestran los porcentajes de evaluación según los ítems: si suficientes, si apropiadas (verde); no suficientes, no apropiadas (morado); si suficientes, no apropiadas (azul claro); no suficientes, si apropiadas (naranja); si apropiadas (azul oscuro); si suficientes (vino tinto); y no suficientes (verde oscuro).

Según estos parámetros de evaluación se evidencia que las metodologías aplicadas en 6 de las 13 materias de especialización (Salud Ocupacional, Seguridad y Salud en el trabajo; Innovación y Tecnología; Gestión de Costos y Métodos de Inventarios; Modelos Operacionales; y Gerencia Estratégica de Producción y Productividad) son evaluadas entre el $80 \%$ y el $97 \%$ de estudiantes como suficientes y apropiadas, solo en un bajo porcentaje (del 3 al 15\%) los estudiantes indican que no son suficientes, pero si apropiadas. Esto indica que más del $80 \%$ de estudiantes, en estas 6 materias adquirieron el conocimiento esperado según el objetivo de aprendizaje planteado por cada materia y están en capacidad de aplicarlo.

Hay 4 de las 13 materias que entre el $67 \%$ y el $78 \%$ de los estudiantes perciben que son suficientes y apropiadas (Gerencia de Producción y Calidad; Juego Gerencial; Robótica y Telecomunicaciones; y Gerencia de Recursos Humanos). Solo en 2 de estas materias entre el 13 y el $24 \%$ de los estudiantes consideran que no son suficientes, ni apropiadas, entre el 4 y el $18 \%$ piensan que no son suficientes, pero si apropiadas y entre el 11 y el $22 \%$ que no son suficientes. Esto indica que más del $67 \%$ de estudiantes cumplieron con los objetivos de aprendizaje planteados y están en capacidad de aplicarlos, sin embargo, el 33\% de los estudiantes sugiere revisar como tal las metodologías aplicadas en estas materias para mejorar su percepción.

Solo para 3 de las 13 materias, entre el 13 y el $82 \%$ de estudiantes perciben que no son suficientes, ni apropiadas las metodologías (Sistematización de la Producción; Informática Industrial; y Metodología de la Investigación). Esto indica que posiblemente hay que hacer cambios sustanciales para implementar metodologías de enseñanza-aprendizaje más acordes con los objetivos de aprendizaje 
de estas materias, ya que los estudiantes opinan para la materia de Metodología de la Investigación que no se dio la orientación adecuada para cumplir con el objetivo de la materia y faltó interés por parte del docente y en informática industrial que faltó práctica en el software y dinamismo en clase.

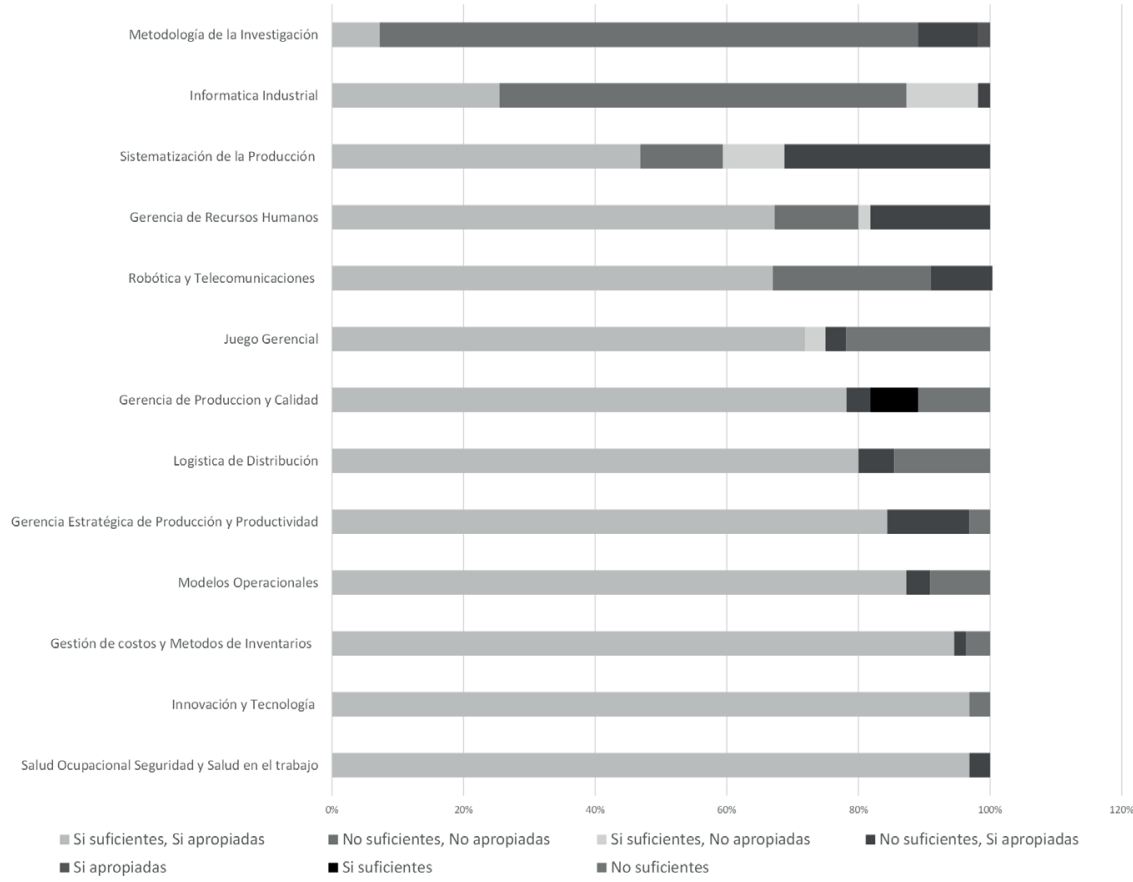

Figura 1. Evaluación de las metodologías según su suficiencia y apropiación para adquirir y aplicar el conocimiento de cada materia.

Finalmente se evaluó con los estudiantes si actualmente son aplicados los conocimientos adquiridos en las materias de la especialización, en la gráfica 2 se puede evidenciar que más del $70 \%$ de estudiantes aplica los conocimientos de 7 de las materias (Gerencia Estratégica de Producción y Productividad, y Juego Gerencial son aplicados por el 93\% de los estudiantes; Gerencia de Producción y Calidad, y Salud Ocupacional, Seguridad y Salud en el Trabajo por el 87\%; Gerencia de Recursos Humanos por el 81\%; Gestión de Costos y Métodos de Inventarios por el 75\%; y Logística de Distribución por el $74 \%)$. 
Entre el $40 \%$ y el $69 \%$ de estudiantes aplican los conocimientos de 3 de las materias (Innovación y Tecnología con el 63\%, Modelos Operacionales con el 58\% y Robótica y Telecomunicaciones con el $57 \%$. Solo en 3 de las materias de la especialización la aplicación de los conocimientos es menor al $40 \%$ de los estudiantes (Sistematización de la Producción con el 37\%; Metodología de la Investigación con el 36\%; e Informática Industrial con el 34\%).

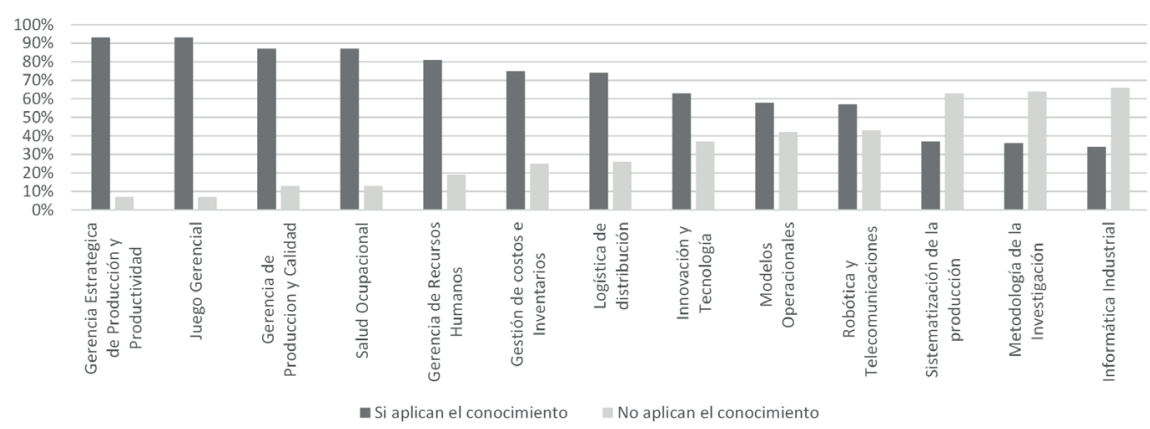

Figura 2. Porcentaje de alumnos que aplican o no aplican actualmente el conocimiento recibido de cada materia.

Los alumnos que si aplican el conocimiento lo hacen en su vida laboral como desempeño en su área y los que no lo aplican es porque no está relacionado con el campo o área que ellos manejan o no han tenido aun la oportunidad de aplicarlos. La opinión de algunos estudiantes en las materias que tienen un bajo nivel de aplicabilidad son las siguientes: Sistematización de la Producción: Opinan que no es el campo laboral de ellos y que no han tenido la oportunidad de aplicarlos; Informática Industrial, dicen que en esta materia se presentaron los conocimientos básicos, no se profundizo, la información fue insuficiente, no hubo práctica, por lo cual no se sienten en capacidad para aplicarla en el campo laboral; Metodología de la Investigación: Opinaron que no se adquirieron los conocimientos suficientes para su aplicación.

Según los datos planteados en la Tabla 2, la Gráfica 1 y la Gráfica 2 , se puede concluir que las metodologías de aprendizaje implementadas en el $62 \%$ de las materias son evaluadas como buenas y excelentes con una nota promedio de 4,1 sobre 5 , estas me- 
todologías son evaluadas por los estudiantes como suficientes y apropiadas en promedio por el $85 \%$ de los estudiantes, y los conocimientos son aplicados en promedio por el $77 \%$ de los estudiantes encuestados. Las metodologías de aprendizaje implementadas en el $23 \%$ de las materias son evaluadas como aceptables con una nota promedio de 3,5 sobre 5, estas metodologías son evaluadas por los estudiantes como suficientes y apropiadas en promedio por el $62 \%$ de los estudiantes, y los conocimientos son aplicados en promedio por el $62 \%$ de los estudiantes encuestados. Y finalmente, las metodologías de aprendizaje implementadas en el $15 \%$ de las materias son evaluadas como regulares con una nota promedio de 2,7 sobre 5, estas metodologías son evaluadas por los estudiantes como suficientes y apropiadas en promedio por el $16 \%$ de los estudiantes, y los conocimientos son aplicados en promedio por el $35 \%$ de los estudiantes encuestados.

\section{Discusión}

Existen diferentes estudios relacionados con la percepción de los estudiantes sobre las metodologías de enseñanza aprendizaje que muestran, que conocer la opinión de los estudiantes es importante para lograr cambios en las metodologías de Enseñanza - Aprendizaje e innovaciones docentes; y de esta manera contribuir para que los estudiantes adquieran un aprendizaje significativo.

Para que haya un aprendizaje significativo por parte de los estudiantes "es necesario conocer la situación de los alumnos antes de empezar cualquier programación, para partir de aquello que ya se sabe y usarlo para conectar y relacionar con los nuevos aprendizajes" (Psicopedagogía, 2015). De esta manera se construirá el aprendizaje en los estudiantes y tendrán las herramientas para aplicarlo ya que el conocimiento se vuelve próximo a la realidad de los estudiantes, de lo contrario "el aprendizaje es básicamente por repetición y se ve sometido rápidamente al olvido" (Psicopedagogía, 2015). 
En el caso de la especialización los docentes manejan principalmente las metodologías de aprendizaje de la Clase Magistral, el aprendizaje cooperativo y el estudio de casos. Estas metodologías los estudiantes las evalúan como aceptables, y en el $77 \%$ de las materias los conocimientos son aplicados por los estudiantes. Solo 3 materias manejan las metodologías inadecuadas que no permiten que el aprendizaje de los estudiantes sea significativo y les permita aplicarlo.

Los opositores de la Clase Magistral, usada por el 100\% de docentes de la especialización como metodología de enseñanzaaprendizaje argumentan que se usa generalmente por "la comodidad que proporciona a los docentes $y$, especialmente a la institución (por el ahorro de recursos que suponen, al facilitar la atención a grupos numerosos de alumnos) que por los resultados de aprendizaje conseguidos" (Ropero, García, \& Diez, 2013). Sin embargo, según la percepción de los estudiantes de la especialización en un $62 \%$ de las materias la evaluación de esta metodología fue buena y excelente, en un $23 \%$ de las materias aceptable y solo en un $15 \%$ regulares. Por otro lado, según un estudio realizado en 3 Universidades del País en programas de Ingeniería, esta metodología fue evaluada entre Excelente y Buena (Garzón, Beltrán, \& Martínez, 2009).

Según los resultados anteriores, a pesar de las críticas a esta metodología es importante emplearla porque el docente necesita exponer de alguna manera los conceptos teóricos que fundamentan la materia, esto lo confirma Verona (2006, p. 94), validando que es un método que en determinadas circunstancias es muy eficaz en el proceso de enseñanza por su facilidad para la transmisión de información, también por ser un método flexible que puede combinarse con otros métodos y por no necesitar medios complejos para ser implementado. Sin embargo, es importante revisar el tiempo que se le dedica a la metodología ya que los alumnos prestan atención los primeros minutos de la exposición y luego se pueden tornar cansados y aburridos; además, el docente debe poseer ciertas cualidades didácticas para poder mantener al grupo concentrado en su exposición. 
Es importante continuar con el uso de la metodología de aprendizaje cooperativo empleada por el $77 \%$ de docentes y evaluada por los estudiantes como aceptable, ya que desde el "punto de vista pedagógico, lo central en la búsqueda de la interacción cooperativa no es que los estudiantes realicen algo juntos, si no que juntos logren aprender" (Suárez, 2010). De la misma manera la metodología de Estudio de Casos (MC), utilizada por el 54\% de los docentes y evaluada como aceptable por los estudiantes es importante aplicarla porque desarrolla la capacidad de análisis y razonamiento, fomenta la capacidad de toma de decisiones, y favorece el trabajo en equipo (García \& Galicia, 2014).

Hay que tener en cuenta que no todas las metodologías aplican a todas las materias, ni tampoco a todo tipo de estudiantes, Verona (2006) afirma que:

Cada método de enseñanza tiene ventajas e inconvenientes, ninguno es plenamente satisfactorio ni suficiente para todos los alumnos ni para todos los objetivos. Al contrario, se puede incluso decir que cada método está indicado preferentemente para un tipo de objetivos, para un tipo de alumnos; requiriendo destrezas y requisitos específicos para poder ser aplicados correctamente. ( $p$. 92)

Esto se evidencia en los resultados de percepción de las metodologías empleadas en la especialización, sin embargo, es importante prestar atención a las metodologías implementadas para los cursos de informática industrial y metodología de la investigación que fueron evaluados como regular en sus metodologías y como no suficientes ni apropiadas, para mejorar la percepción y el aprendizaje de los estudiantes.

En el caso de las metodologías usadas en menor porcentaje en las materias de la especialización como Aprendizaje Basado en Proyectos, Aprendizaje basado en problemas y Simulación y juego revisar su aplicación en todas las materias ya que en el caso de la metodología del Aprendizaje Basado en Proyectos integra lo aca- 
démico, lo laboral e investigativo y favorece "la identificación de problemas profesionales, generación de alternativas y aplicación de métodos tecnológicos inherentes a los procesos básicos de la carrera universitaria en que se trabaje" (Tejada \& Sánchez, 2009). Lo anterior hace que el conocimiento sea aplicado a situaciones reales y que además se integren diferentes disciplinas del conocimiento, lo cual hace que el aprendizaje sea significativo e integrador del conocimiento (Ferreyra, 2014).

Por otro lado, teniendo en cuenta que al implantar las metodologías del Aprendizaje Basado en Problemas (ABP) y el Método de Casos, se obtiene que el conocimiento aumente considerablemente en todos los alumnos, según Diez \& IPDDA (2010), es importante que se promuevan en mayor medida estas metodologías que son activas y que en el caso del Aprendizaje Basado en Problemas solo se usa por el $15 \%$ de los docentes de la especialización. O manejar también la metodología de Aprendizaje Basado en Proyectos y estudio de casos que dieron un muy buen resultado para la materia Gerencia de Producción y Calidad de la especialización por usar metodologías suficientes y apropiadas y tener una evaluación superior a 4 (Ver tabla 3).

En el caso de la metodología de Simulación y Juego solo utilizada por 2 profesores de la especialización y evaluada como buena y excelente en una materia y aceptable por otra, se puede considerar usarla en otras materias ya que permite simular problemas de la realidad a los que los estudiantes se pueden ver enfrentados para hallar las soluciones (Andreu, García, \& Mollar, 2005). De la misma manera se debe indagar sobre las metodologías que no son utilizados por los docentes de la especialización: Aprendizaje Basado en Procesos, que contribuye a un cambio integral en el alumno y Contrato de Aprendizaje, que desarrolla habilidades comunicativas, interpersonales y organizativas.

Finalmente, se debe entender que la medición de la calidad de los procesos de enseñanza aprendizaje es un tema difícil y muy grande de abarcar "porque la percepción de los seres humanos es muy compleja y subjetiva" (Rainer, 1986), sin embargo, a pesar de 
la complejidad y subjetividad de la percepción de los estudiantes, son un insumo importante a tener en cuenta para que los docentes autoevalúen su desempeño en la generación de aprendizaje en los estudiantes y en verificar si sus métodos son los adecuados para que se construya el aprendizaje en ellos.

\section{Conclusiones}

Según la percepción de los estudiantes respecto a las metodologías de enseñanza-aprendizaje empleadas en cada materia (13) según los objetivos de aprendizaje planteados, la evaluación de las metodologías según su suficiencia y apropiación para adquirir y aplicar el conocimiento de cada materia y la validación de la aplicación del conocimiento aprendido en cada materia de la especialización en Gerencia de Producción y Productividad, se concluye que hay una estrecha relación entre la evaluación de las metodologías de aprendizaje impartidas por los docentes y la adquisición y aplicación del conocimiento aprendido por los estudiantes. Esto se evidencia en el $62 \%$ de las metodologías de las materias que fueron muy bien evaluadas y que son aplicadas por el $77 \%$ de estudiantes encuestados. Por el contrario, del 15\% de las metodologías de las materias que fueron evaluadas como regulares, solo el $35 \%$ de los estudiantes aplica estos conocimientos. Según lo anterior es pertinente que haya una autoevaluación del docente respecto a los resultados de la evaluación de los estudiantes entorno a las metodologías de las materias de la especialización para indagar cuales son las metodologías adecuadas que permitirán que el estudiante adquiera el conocimiento y esté en capacidad para aplicarlo.

Por otro lado, es importante tener en cuenta los tiempos del semestre para impartir contenidos adecuados a esta disponibilidad y no intentar abarca gran cantidad de contenidos que a la final no van a ser de utilidad para los alumnos por no poderlos procesar adecuadamente. Ya que la evaluación dada por los estudiantes en las metodologías que no son suficientes y adecuadas esta fue una observación reiterativa. Este es un factor clave si se 
quiere promover un aprendizaje significativo para los estudiantes. Por este motivo teniendo en cuenta que los programas académicos son relativamente cortos se debe realizar un análisis del contenido general de los programas presentado en cada materia por los docentes y seleccionar los temas que son importantes y relevantes para la especialización en forma interdisciplinar para que los temas no se repitan. Además, es transcendental tener en cuenta el rol laboral desempeñado por los estudiantes de la especialización ya que muchos de los que aplican el conocimiento adquirido en la especialización es porque hay una relación directa con las materias y su trabajo.

La calidad de la educación implica la aplicación de las teorías educativas y metodologías de enseñanza aprendizaje de los docentes en el aula. En este sentido es necesario que los docentes tengan conciencia del trabajo que realizan, haciendo necesario para ellos talleres y seminarios que les permita conocer las teorías educativas y la innovación en metodologías de enseñanza aprendizaje. Para así hacer conciencia de los procesos formativos y de una mejora continua de los procesos. Debe impulsarse a los docentes en el uso de Metodologías de Aprendizaje activas como son: Aprendizaje Basado en Proyectos (ABP); Aprendizaje Basado en Problemas (ABP) y Estudio de Casos para que además de tener metodologías de aprendizaje suficientes y apropiadas, éstas contribuyan a adquirir un aprendizaje significativo. Estas metodologías contribuyen a adquirir un aprendizaje significativo porque se caracterizan por presentarse en contextos de la vida real y de la práctica profesional, en el cual el estudiante se desarrolla o desarrollará en el futuro. Y aunque son las menos aplicadas en la especialización, fueron las mejor evaluadas por los estudiantes.

\section{Recomendaciones}

- Se recomienda que los docentes diversifiquen el uso de las metodologías en la materia que imparten y revisen cuales son las más adecuadas para que los estudiantes adquieran un aprendizaje significativo. 
- Se recomienda capacitar a los docentes en metodologías de aprendizaje para que puedan tener conocimiento de diferentes metodologías y aplicar las más adecuadas a su materia.

- Se recomienda que haya más integración entre la materia de Metodología de Investigación y el proyecto de grado. Esta materia debería ser un apoyo durante todo el proyecto y calificarse de manera Individual.

\section{Referencias bibliográficas}

Andreu, M., García, M. y Mollar, M. (2005). La simulación y juego en la enseñanza-aprendizaje de lengua extranjera. Cuadernos Cervantes, XI (55), pp. 34-38.

Aparicio, O.Y. (2018). La investigación escolar. Revista Interamericana de Investigación, Educación y Pedagogía, RIIEP, 11(2). https://orcid. org/0000-0003-3535-6288

Aparicio, O.Y., \& Ostos, O.L. (2018). El constructivismo y el construccionismo. Revista Interamericana de Investigación, Educación y Pedagogía, RIIEP, 11(2). https://orcid.org/0000-0003-3535-6288. https://orcid.org/0000-0002-6477-9872

Coll, C. (1987). Capítulo II, Fundamentos del currículum, en psicología y Currículum, México, Paidós, pp 21-47.

Diez, M., \& IPDDA, G. (2010). Percepción de metodologías docentes y desarrollo de competencias al EEES. Boletín de Psicología, No 99, 45-69.

de Almeida, R. R., Santos, M. F., \& Porto, J. C. (2017). Lectura de textos ficcionales y el enfoque escolar de literatura: contribuciones para una Pedagogía de la Elección. Revista Interamericana de Educación, Pedagogía y Estudios Culturales, 9(1), 35-51. DOI: https://doi. org/10.22490/25391887.1925

De Miguel, M. (2006). Metodologías de enseñanza y aprendizaje para el desarrollo de competencias. Orientaciones para el profesorado 
Universitario ante el espacio europeo de educación superior. Madrid: Alianza Editorial.

Ferreyra, H. A. (2014). Mesas Socioeducativas para la Inclusión y la Igualdad. Un programa "De todos con todos". Una experiencia en construcción. Revista Interamericana de Investigación, Educación y Pedagogía, RIIEP, 7(2). DOI: https://doi.org/10.15332/s1657107X.2014.0002.01

García, F., \& Bagán, F. (2006). Generaliat Valenciana. Recuperado el 30 de Septiembre de 2014, de http://www2.minedu.gob.pe/digesutp/ formacioninicial/wp-descargas/bdigital/028_Contrato_didactico_y_El_ Portafolio.pdf

García, A., \& Galicia, S. (2014). Curso: Ocho metodologías relacionadas con el Arte y la Ciencia de enseñar. Pág. 21. 9no congreso Internacional de Educación Superior. Imprenta del Palacio de convenciones de La Habana: Universitaria.

Garzón, C., Beltrán, L., \& Martínez, P. (2009). Estudio de Percepción sobre Metodologías de Enseñanza de Temas de Electrónica en programas diferentes a Ingeniería Electrónica. Educación en Ingeniería, 98.

Langer, E. (2016). La construcción de confianza para el estudio de prácticas de resistencia en la escolarización de jóvenes en contextos de pobreza urbana. Revista Interamericana de Investigación, Educación y Pedagogía, RIIEP, 9(2). DOI: https://doi.org/10.22490/25391887.1945

Maldonado, M. (2008). Aprendizaje Basado en Proyectos Colaborativos. Una experiencia en educación superior. 14(28), 160-163

Martin, E. (2003). Aportaciones de Piaget Suare a la teoría y prácticas educativas. España: Colegio Oficial de Psicólogos de Madrid.

Núñez, G., I. (2015). Aprendizaje de competencias versus aprendizaje de contenidos científicos. Tesis doctoral. Pág. 83 -85. Universidad de Granada, Granada.

Ortiz, J. G., \& Buitrago, H. (2017). La evaluación en la tradición educativa 
colombiana. Instrumento de clasificación social. Revista Interamericana de Investigación, Educación y Pedagogía, RIIEP, 10(1), 145-171. DOI: https://doi.org/10.15332/s1657-107X.2148

Pérez, A. (2014). Metodologías de enseñanza y aprendizaje para el desarrollo de competencias. Pág. 22. Madrid. Difusora Larousse - Alianza Editorial.

Psicopedagogía (2015). Artículo psicología de las asignaturas. Madrid, España.; Psicopedagogía, Psicología de la educación para padres y educadores. Recuperado de http://www.psicopedagogia.com/ articulos/?articulo $=367$

Rainer, G. (1986). Diseño Psicológico de la información humana. Biblioteca de Psicología 172.

Ropero, J., García, B., \& Diez, M. (2013). Claves para la aplicación de nuevas metodologías docentes en derecho inspiradas en el proceso de Bolonia. Madrid: Dykinson.

Suárez, C. (2010). Cooperación como condición social de aprendizaje. España: Editorial UOC.

Tejada, R., \& Sánchez, P. (2009). Los proyectos y su contribución en la formación competente de profesionales universitarios. Rev. Pedagogía Universitaria [Internet]. 2009; 14 (1). Acceso. http://cvi.mes.edu.cu/ peduniv/index.php/peduniv/article/view/483/477

Verona, M., C. (2004). Métodos didácticos aplicables a materias de las disciplinas administrativas. De la lección magisterial al campus virtual. Tiempo de Educar, vol. 5, núm. 9, enero-junio, 2004, pp. 89-114 Universidad Autónoma del Estado de México Toluca, México. Disponible en http://www.redalyc.org/pdf/311/31100904.pdf

Zarate, J. (2002). El arte de la relación maestro alumno en el concepto enseñanza aprendizaje. Pág. 19. México: Instituto Politécnico Nacional.

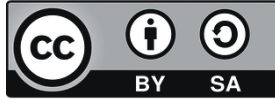

